\title{
Contrasting effects of sensory limits and capacity limits in visual selective attention
}

\author{
NILLI LAVIE \\ University College London, London, England \\ and \\ JAN W. DE FOCKERT \\ Goldsmiths College, University of London, London, England
}

\begin{abstract}
The effects of perceptual load and those of target-stimulus degradation on distractor processing were contrasted. Targets either had to be found among several nontargets (high perceptual load) or were presented alone and were intact (low perceptual load), had reduced size and contrast (Experiment 1), had reduced duration and were followed by a mask (Experiment 2), or had reduced visual acuity owing to position eccentricity (Experiment 3) in the degraded low-load condition. The results showed that both high perceptual load and target degradation increased general task difficulty, as is reflected by overall reaction times and accuracy. However, whereas high perceptual load reduced response-competition effects of irrelevant distractors, target degradation increased distractor effects. These results support the hypothesis that distractor processing depends on the extent to which high perceptual load exhausts attention in relevant processing, and provide a dissociation between perceptual load and general task difficulty and processing speed.
\end{abstract}

Although people may sometimes succeed in ignoring irrelevant information, this is not always the case, and irrelevant distractors are often found to produce interference effects on performance (see, e.g., B. A. Eriksen \& C. W. Eriksen, 1974; Gatti \& Egeth, 1978). Indeed, the extent to which irrelevant distractors can be successfully ignored, to the point of exclusion from perception, has been an issue of intensive debate for several decades of selective attention research (for a review, see Lavie \& Tsal, 1994). Studies that demonstrated apparently successful exclusion of distractors from perception (e.g., Francolini \& Egeth, 1980; Yantis \& Johnston, 1990) have led to an early selection view (advanced by Broadbent, 1958; Treisman \& Geffen, 1967; see also Yantis \& Johnston, 1990), in which attention can prevent distractor perception. However, numerous reports of people's failure to ignore irrelevant distractors have accumulated as well (e.g., Driver \& Tipper, 1989; Miller, 1991; for a review, see Lavie \& Tsal, 1994) and have led to an opposing late selection view (advanced by Deutsch \& Deutsch, 1963, 1967; also expressed in more recent theories, e.g., Duncan, 1980, and Tipper, 1985), in which distractors can be excluded only from postperceptual processes (such as memory and responding), but their perception cannot be prevented.

This research was supported by a Medical Research Council (U.K.) career award and by Biotechnology and Biological Sciences Research Council (U.K.) Grant 31/S09509 to the first author. Address correspondence to N. Lavie, Department of Psychology, University College London, Gower St., London WC1E 6BT, England (e-mail: n.lavie@ ucl.ac.uk).
These conflicting results can be accommodated by hybrid models of attention, in which selective attention can result in successful exclusion of distractors from perception (i.e., early selection) in some circumstances, and in a failure to exclude distractors from perception (i.e., late selection) in others. Lavie (1995, 2000, 2001; Lavie \& Tsal, 1994) has recently suggested such a hybrid model, in which the level of perceptual load of the relevant processing determines whether early selection or late selection occurs. Early selection occurs under situations of high perceptual load (e.g., when many relevant stimuli are presented) that exhaust all available capacity in relevant perception. Late selection occurs under situations of low perceptual load (e.g., just one relevant stimulus is presented), because relevant perception leaves spare capacity that "spills over" to the processing of irrelevant items.

Empirical support for the role of perceptual load in determining the processing of irrelevant distractors has been provided in a series of experiments that manipulated the level of perceptual load in relevant processing and measured the effects on irrelevant distractor processing. These studies used various manipulations of perceptual load and several measures of distractor processing (for a review, see Lavie, 2000, 2001). For example, Lavie (1995) and Lavie and Cox (1997) manipulated perceptual load by varying the number of stimuli among which targets had to be found (i.e., the relevant search set size; see also Lavie, 1995 ) or by varying the similarity between target and nontarget letters in a task of visual search. In both studies, distractor processing was assessed through the responsecompetition effects it produced on target reaction times 
(RTs). The results from these studies showed that responsecompetition effects from peripheral irrelevant distractors were reduced by high perceptual load in the relevant processing. Lavie and Fox (2000) have recently examined the effects of perceptual load on negative priming from an irrelevant distractor. They found that the extent to which an irrelevant distractor in the prime display produced negative priming effects on subsequent target RTs was critically determined by the number of task-relevant stimuli in the prime displays.

Perceptual load has also been manipulated by varying the processing requirements for the same stimuli. For instance, Lavie (1995) demonstrated that distractor-responsecompetition effects were reduced when target selection required the processing of a conjunction of features rather than simple feature registration (a definition of load provided by feature integration theory of attention; Treisman \& Gelade, 1980), or precise discrimination rather than simple presence-versus-absence detection (see Bonnel, Possamai, \& Schmitt, 1987; Graham, 1989; Uttal, 1987). Rees, Frith, and Lavie (1997) found that neural responses to motion distractors in sensory areas responsive to motion (e.g., V5) were reduced when a relevant task involved more complex linguistic discrimination rather than simple detection of letter case.

Thus, a variety of conventionaloperationaldefinitions of load, which have varied either the number of stimuli or their processing requirements, all converged to show that distractor processing is reduced by high perceptual load. However, a single, overarching definition of perceptual load has not yet been provided. Moreover, perceptual load typically increases general task difficulty and results in longer overall RTs and reduced accuracy (but see Lavie \& Fox, 2000 , for an exception). Thus, the effects of load in at least some of the previous perceptual load studies (i.e., those in which RT has been used to measure distractor processing and which have shown that, in addition to reducing distractor effects, load has also significantly increased overall RT) may be attributed to an increase in general task difficulty and general slowing of responses with load, rather than being specific effects of load on perceptual resources. For example, in the response-competition studies described earlier, distractors may have been processed after all, but their effects on RTs may have dissipated by the time a response was made. The purposes of the present study were to further clarify the concept of perceptual load and to dissociate the effects of perceptual load from the general effects of task difficulty and processing speed.

The concept of perceptual load implies that additional perceptual operations must be carried out under higher load, or that the same operations must be applied to more items. It is these items or operations that consume attentional capacity in the relevant processing, thereby blocking irrelevant processing. However, unlike increases in perceptual load, making a task harder without adding further demands on attentional capacity should not result in selective processing. For example, degrading the sensory quality of a target stimulus (e.g., by reducing its contrast) should make the task of identifying the target harder, but this will not necessarily increase the demand on attentional capacity. For example, if a target stimulus is degraded so severely that it becomes invisible, any further allocation of attention will not improve its perception. Here, we are using a distinction, first suggested by Norman and Bobrow (1975), between two different kinds of processing limitations: data limits on the quality of the input to the perceptual system, and resource limits on the processing of the input. Norman and Bobrow further assumed that data limits on input cannot be compensated for by applying more resources to processing. Thus, according to the perceptual load hypothesis, a manipulation of sensory degradation of the target stimulus that induces data limits rather than resource limits should increase general task difficulty, but it should not decrease distractor processing, given that the latter depends on the availability of attentional resources. Only a manipulation that imposes high load on attentional resources should reduce distractor effects.

To test this hypothesis, we contrasted the effects of perceptual load on distractor processing with the effects of various manipulations of sensory degradation of the target stimulus. Subjects performed a variation of a responsecompetition task, in which they made speeded choices in response to a central target, while attempting to ignore an irrelevant distractor in the periphery. This peripheral distractor was either compatible or incompatible with the target response. Perceptual load was manipulated in this task by variation of the number of relevant nontarget letters in the center of the display (see Lavie \& Cox, 1997). That is, the targets were presented either alone (in the low-perceptualload condition) or accompanied by a number of nontargets in the center of the display (in the high-perceptual-load condition). The new condition of interest was that of low perceptual load with a degraded target. In this condition, the target was presented alone, but target size and contrast were reduced in comparison with those used in the other two conditions (Experiment 1), target contrast and presentation duration were reduced and the target was followed by a mask (Experiment 2), or target eccentricity was varied (through variations in target position) to produce more peripheral targets with reduced retinal acuity (Experiment 3 ).

We anticipated that all the conditions with a degraded target stimulus would result in increased task difficulty and longer RTs, but not in reduced distractor processing. Manipulations of sensory degradation were expected to induce data limits rather than resource limits; thus, target processing in these conditions was expected to leave spare attention that would "spill over" to irrelevant processing. On the other hand, because high perceptual load should engage attention in relevant processing, it was expected to reduce irrelevant distractor processing. Thus, only in conditions of high perceptual load did we expect smaller distractor-interference effects in comparison with lowperceptual-load conditions, with both intact targets and degraded targets. 

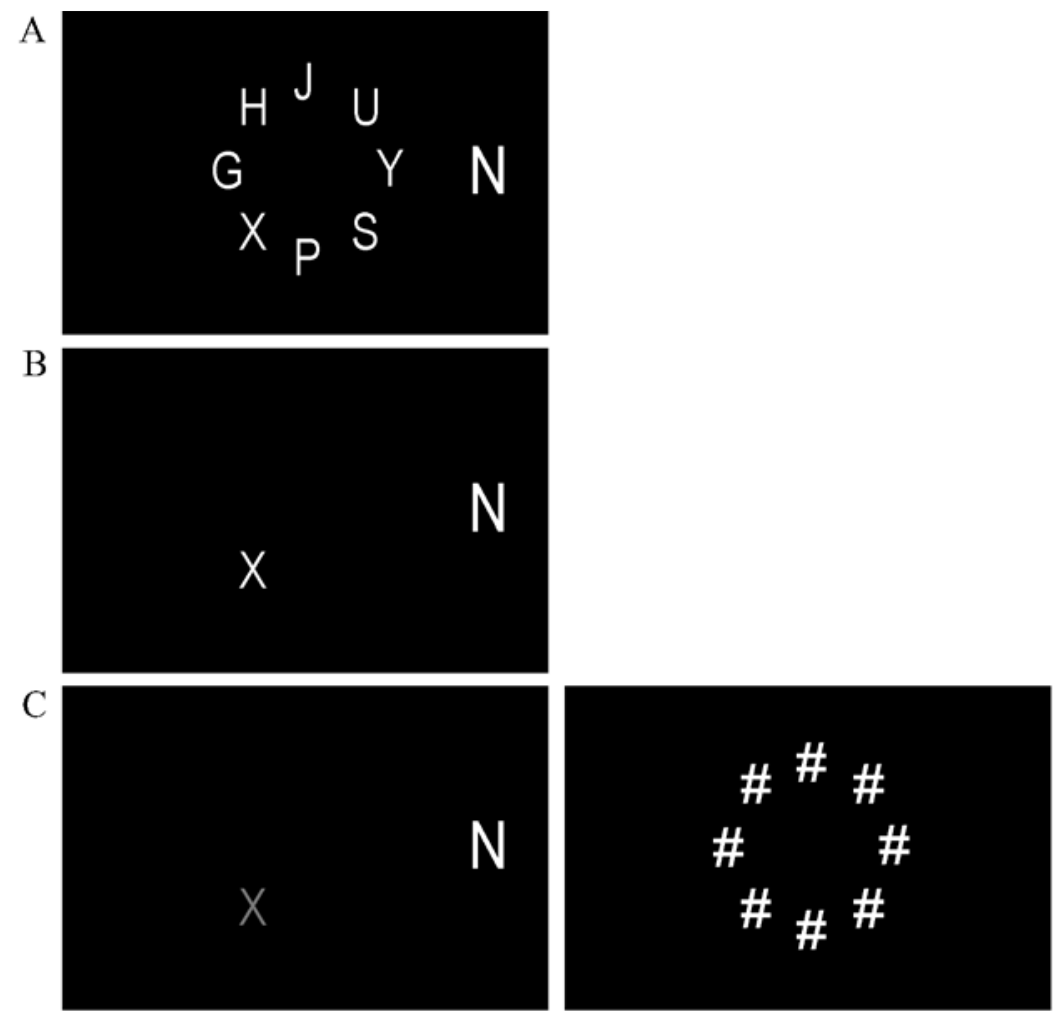

Figure 1. Examples of displays with an incompatible distractor for conditions of high perceptual load (A), low perceptual load (B), and degraded low perceptual load (C) used in Experiments 1 and 2. In Experiment 1, no mask was presented, and the size of the target was reduced.

\section{EXPERIMENT 1}

In Figure 1, examples of stimulus displays used in Experiments 1 and 2 are presented. The purpose of Experiment 1 was to contrast the effects on distractor processing of perceptual load and target-stimulus degradation. Perceptual load was manipulated by varying the relevant set size, whereas target-stimulus degradation was achieved by reducing the size and intensity of the target stimuli. A central target was presented in one of eight circle positions chosen randomly on each trial. In addition, an irrelevant distractor was presented in the left or right periphery. The nontarget positions in the circle either remained empty (in the conditions of low perceptual load) or were occupied with seven neutral letters that served to load the search for the target (in the condition of high perceptual load). These nontarget letters were response neutral, so that the manipulation of set size did not vary the number of response alternatives. Moreover, we ensured that the spacing between individual letters in the circle was sufficient to preclude effects of lateral masking or crowding (Huckauf, Heller, \& Nazir, 1999; Strasburger, Harvey, \& Rentschler, 1991; see also Wolford \& Chambers, 1983). In the condition of low perceptual load with degraded targets, the target letter was approximately half the size that it was in the other conditions, and the contrast intensity of the target stimulus was substantially reduced.

We predicted that both conditions of high perceptual load and low perceptual load with degraded targets would increase task difficulty, resulting in longer overall RTs and reduced accuracy. Distractor-response-competition effects, however, were expected to be reduced only in the high-load condition, in comparison with conditions of low perceptual load with intact targets as well as with degraded targets.

\section{Method}

Subjects. Sixteen students from the subject pool of the Medical Research Council-Applied Psychology Unit in Cambridge participated. All the subjects had normal or corrected-to-normal vision and were paid for their participation.

Apparatus and Stimuli. An IBM-compatible computer running Micro Experimental Laboratory software (MEL; Schneider, 1988) connected to a 15-in. SVGA monitor was used for stimulus presentation and data collection. Viewing distance was held fixed at $60 \mathrm{~cm}$ with a custom-built hood. Each display consisted of a target letter (either $\mathrm{N}$ or $\mathrm{X}$ ) subtending $0.9^{\circ}$ of visual angle vertically $\times 0.7^{\circ}$ horizontally in the low-load and high-load conditions, and $0.31^{\circ} \times 0.3^{\circ}$ in the degraded low-load condition. An irrelevant distractor subtending $0.9^{\circ}$ of visual angle vertically $\times 0.7^{\circ}$ horizontally was also present on each display. A black background was used, and all stimuli were presented in light gray (color 7 in the MEL color palette), 
except for the target letter in the low-load condition with degraded targets, which was presented in dark gray (color 56 in the MEL color palette). The target letter was presented at random in one of eight possible locations arranged in a circle centered at $1.3^{\circ}$ from fixation. In all low-load displays, the target letter was presented alone in the circle, and the other seven locations remained empty. In high-load displays, the other seven locations were occupied by nontarget letters $(\mathrm{G}, \mathrm{H}, \mathrm{J}, \mathrm{P}, \mathrm{S}, \mathrm{U}$, and Y), which were of the same size as the target. These nontargets appeared equally often, and their positions were randomly chosen for each display. The center-to-center distance between the letters in the circular array was $0.95^{\circ}$. The distractor was presented either to the left or to the right of the circle of letters, at $3.2^{\circ}$ from fixation, and was equally likely to be the same letter as the target for that display (compatible condition), the target letter that was not presented in the current display (incompatible condition), or the nontarget letter $\mathrm{P}$ (neutral condition). The various conditions of target identity, target position, distractor identity, and distractor position occurred equally often in each block, as did all combinations of these factors. For each task condition, 72 different displays were created according to these specifications.

Procedure. Each trial began with a fixation display presented for $1 \mathrm{sec}$, which consisted of eight dots in a circular arrangement, marking the eight possible target locations. Next, the stimulus display appeared for $100 \mathrm{msec}$. The subjects were instructed to identify as rapidly and accurately as possible whether the target letter in the relevant set was an $\mathrm{N}$ or an X by pressing " 0 " on the numeric computer keypad for $\mathrm{N}$ and " 2 " for $\mathrm{X}$, while ignoring the peripheral distractor letter. Feedback for errors was given in the form of a $100-\mathrm{msec}$ tone. Following the response, or after a 5-sec response window, the fixation display of the next trial was presented.

Task conditions (low load, high load, and degraded low load) were blocked. For each task condition, the subjects performed 4 successive blocks of 72 trials, preceded by a practice block of 20 trials. Thus, there were 12 experimental blocks. Four of the six possible orders of presentation of task conditions were performed by 3 subjects each, and two of the presentation orders were performed by 2 subjects each.

\section{Results and Discussion}

Mean RTs and error rates were calculated for each subject as a function of the experimental factors (see Figure 2). Trials with incorrect responses or RTs over $2 \mathrm{sec}$ were ex- cluded from the RT analysis. Fewer than $0.1 \%$ of the trials were excluded due to the 2 -sec cut-off criterion.

Effects of perceptual load and stimulus degradation on task difficulty. Within-subjects analyses of variance (ANOVAs) were conducted on the RTs and error rates with the factors of task condition (low load, high load, degraded low load) and distractor compatibility (incompatible, neutral, compatible). These analyses revealed a main effect of task condition on the RTs $[F(2,30)=$ $47.87, p<.001]$, but not on the error rates $[F(2,30)=1.84$, $p=.18]$. Planned contrasts showed that RTs were significantly longer on high-load displays $(M=627 \mathrm{msec})$ than on degraded low-load displays $[M=570 \mathrm{msec}, t(15)=$ $4.43, p<.001]$, which, in turn, produced longer RTs than did low-load displays $[M=507 \mathrm{msec}, t(15)=8.92, p<$ $.001]$. The error data showed a similar trend, increasing from $7 \%$ under low load to $9 \%$ under both high load and degraded low load. Thus, both manipulations of relevant perceptual load and those of target degradation increased general task difficulty and resulted in longer RTs and reduced accuracy in comparison with a condition of low perceptual load with intact targets.

Distractor interference effects. To assess how distractor interference effects were affected by task condition, ANOVA comparisons of the RTs and error rates in incompatible and neutral distractor conditions between task conditions (low load, high load, degraded low load) were conducted. The RT analysis revealed a significant interaction between task condition and distractor compatibility $[F(2,30)=6.36, p<.01]$. Planned contrasts showed that distractor effects were significantly reduced in the highload condition in comparison with the low-load condition $[t(15)=1.82, p<.05$, for the difference $]$ and with the degraded low-load condition $[t(15)=2.96, p<.005$, for the difference; see also Figure 2]. In contrast, distractor interference effects in the degraded low-load condition were in fact significantly greater in comparison with both the high-

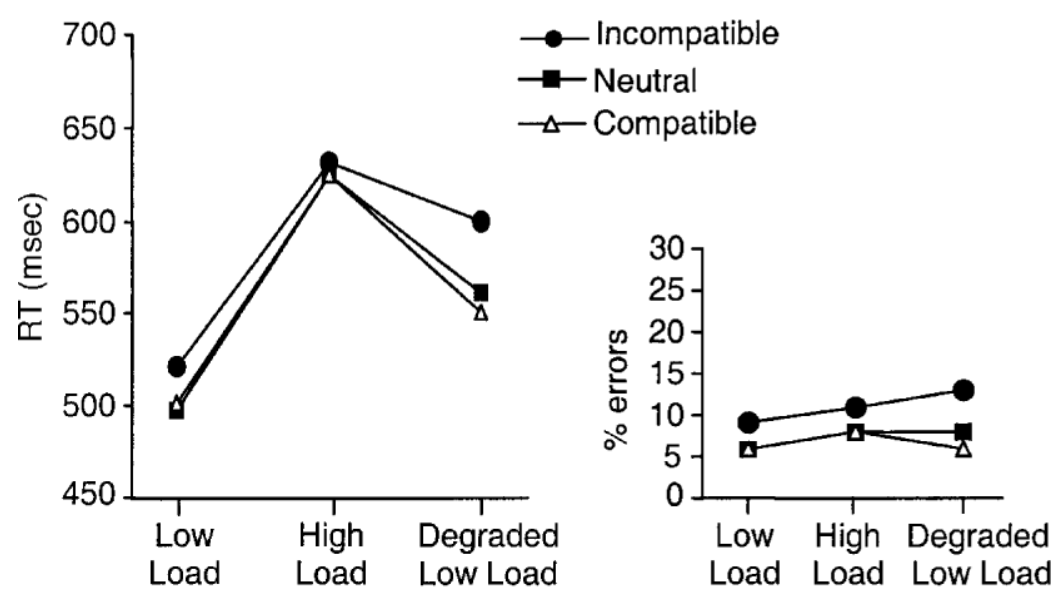

Task Condition

Figure 2. Mean correct-response reaction times (RTs) and error rates as a function of task condition and distractor compatibility in Experiment 1. 
load $[t(15)=2.96, p<.005$, for the difference $]$ and lowload $[t(15)=2.29, p<.025$, for the difference $]$ conditions. Distractor interference effects were significant in conditions of both low load $[M=24 \mathrm{msec}, t(15)=5.91, p<.001]$ and degraded low load $[M=38 \mathrm{msec}, t(15)=8.06, p<$ $.001]$, but not in those of high load $(M=7 \mathrm{msec}, p>.10)$.

Although the error rate analysis failed to reveal a significant interaction $(F<1)$, the numerical trends were similar to those of the RTs (see Figure 2). Distractor effects in the degraded low-load condition $(M=5 \%)$ were numerically greater than the distractor effects in the lowload condition $(M=3 \%)$ and in the high-load $(M=2.5 \%$ interference) condition.

Distractor facilitation effects. In order to establish whether, in addition to the interference produced by incompatible distractors, any facilitation was produced by compatible distractors, ANOVA comparisons of RTs and error rates in compatible and neutral conditions between the conditions of task difficulty (low load, high load, degraded low load) were conducted. In the RT analysis, there was a significant interaction between task condition and distractor compatibility $[F(2,30)=3.29, p=.05]$. As can be seen in Figure 2, facilitation effects were greater in the degraded low-load condition $(M=11 \mathrm{msec})$ than in the high-load condition $(M=0 \mathrm{msec})$ and in the low-load condition $(M=-5 \mathrm{msec})$. However, none of these distractor effects was statistically significant $(p>.10$ in all comparisons). There were no significant main effects or interactions in the error-rate analysis ( $p>.20$ for all effects).

In summary, although both the manipulation of relevant perceptual load and that of target degradation increased general task difficulty, the extent to which irrelevant distractors were processed depended critically on the type of manipulation of task difficulty. Increasing task difficulty by increasing perceptual load led to a reduction in distractor processing, whereas increasing task difficulty by degrading the target stimulus led to an increase in distractor processing.

These results demonstrate that the reduction in distractor interference seen under high perceptual load is not merely a result of a general increase in task difficulty and the associated slowing of responses, but, instead, crucially depends on the extent to which availability of attention is affected by the difficulty manipulation. Clearly, increasing the number of stimuli that need to be identified imposes a greater demand on attention (see, e.g., Treisman, 1991), whereas degrading the target stimulus by reducing its size and contrast seems to have affected task difficulty by inducing data limits rather than resource limits (see, e.g., Norman \& Bobrow, 1975). The finding that stimulus degradation and perceptual load had opposite effects, with stimulus degradation resulting in a significant increase in the processing of irrelevant distractors, is expected in some models. Prolonged processing of the target provides a greater chance of distractor intrusion during the accumulated time (see, e.g., C. W. Eriksen \& Schultz, 1979; Navon, 1989).

We note, however, that although in comparison with low load task difficulty was significantly increased by both the manipulation of perceptual load and that of target-stimulus degradation, the increase in RTs produced by target degradation was smaller than that produced by higher perceptual load. This smaller effect of target degradation on overall RTs cannot explain the opposite effects of perceptual load and target degradation on distractor processing. Nevertheless, it seemed desirable to attempt a manipulation of target degradation that might increase task difficulty and associated RTs to an extent similar to that produced by the manipulation of perceptual load. This was the purpose of the next experiment.

\section{EXPERIMENT 2}

In Experiment 2, we attempted to generalize the effects of target-stimulus degradation to a different manipulation of stimulus degradation. Also, we sought a strong manipulation of sensory stimulus degradation that might increase general difficulty and RTs to a degree similar to that of the increase in difficulty produced by perceptual load. To achieve this, we degraded the target stimulus in the condition of degraded low load by using stimuli of low contrast, reducing the presentation duration of displays to half that of the displays in the other experimental conditions, and presenting masking characters in target positions following the display. We predicted again that only perceptual load should reduce distractor processing. In contrast, the anticipated increase in task difficulty and in RTs in the degraded low-load condition was expected, if anything, to result in an increase in distractor processing, due to the greater time window it provides for distractor intrusions.

\section{Method}

Subjects and Apparatus. Sixteen students from the subject pool of the Medical Research Council-Applied Psychology Unit in Cambridge participated. All the subjects had normal or correctedto-normal vision and were paid for their participation. None had participated in Experiment 1. The apparatus and viewing distance were the same as in Experiment 1.

Stimuli and Procedure. The displays were the same as those used in Experiment 1, apart from the following changes. The target and nontarget letters now subtended $0.6^{\circ}$ vertically $\times 0.4^{\circ}$ horizontally, in all conditions. The distractor letters subtended $1^{\circ}$ vertically $\times$ $0.55^{\circ}$ horizontally. Display duration was reduced to $50 \mathrm{msec}$ in the degraded low-load condition and was followed by a mask consisting of eight "\#" characters, each presented in one of the eight possible target locations. The masking display remained on the screen until a response was made or after $2 \mathrm{sec}$ had elapsed. The target letter in the degraded low-load condition had the same low contrast as in Experiment 1 . The procedure was the same as that used in Experiment 1.

\section{Results and Discussion}

Mean RTs and error rates were calculated for each subject as a function of the experimental factors and are presented in Figure 3. Trials with incorrect responses or RTs over $2 \mathrm{sec}$ were excluded from the RT analysis. Fewer than $0.3 \%$ of the trials were excluded due to the 2 -sec cut-off criterion.

Effects of perceptual load and stimulus degradation on task difficulty. Within-subjects ANOVAs of the RTs and error rates with the factors of task condition (low 

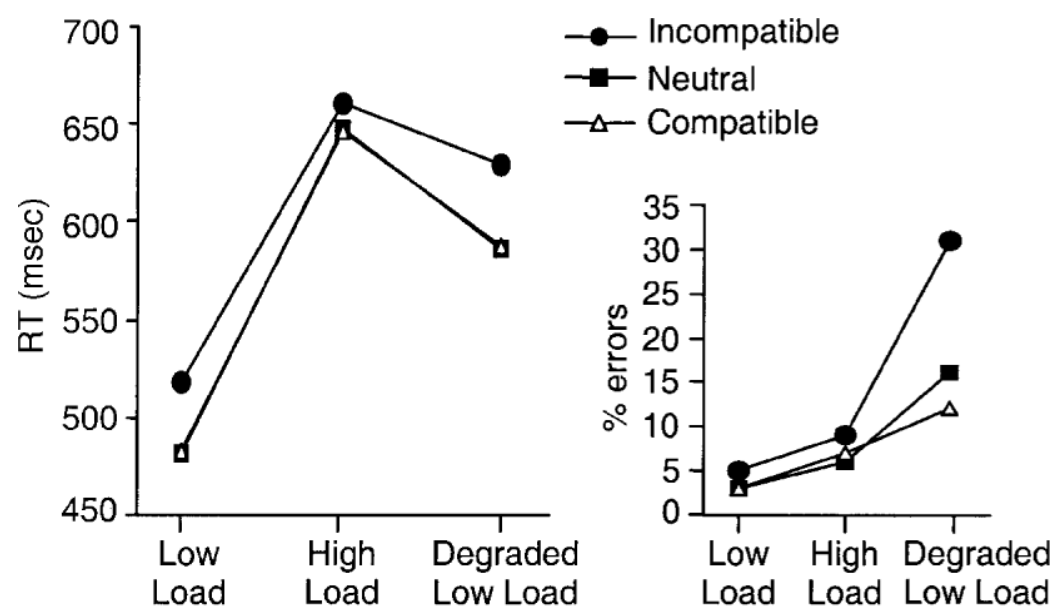

Task Condition

Figure 3. Mean correct-response reaction times (RTs) and error rates as a function of task condition and distractor compatibility in Experiment 2.

load, high load, degraded low load) and distractor compatibility (incompatible, neutral, compatible) revealed a main effect of task condition on RTs $[F(2,30)=16.72, p<$ $.001]$ and error rates $[F(2,30)=24.59, p<.001]$. Planned contrasts showed that RTs in the high-load condition were significantly longer $(M=651 \mathrm{msec})$ than RTs in the degraded low-load condition $[M=601 \mathrm{msec} ; t(15)=1.81$, $p<.05]$, which, in turn, resulted in longer RTs than did the low-load condition $[M=494 \mathrm{msec} ; t(15)=3.72, p<.003$; see also Figure 3]. Error rates, however, were higher in the degraded low-load condition $(M=20 \%)$ than in the highload condition $[M=7 \% ; t(15)=3.97, p<.001$, for the difference], which, in turn, resulted in more errors than did the low-load condition $[M=4 \% ; t(15)=3.71, p<.003$, for the difference].

Distractor interference effects. To assess how distractor interference was affected by task condition, we conducted ANOVA comparisons of RTs and error rates in incompatible and neutral distractor conditions between the task conditions (low load, high load, degraded low load). The RT analysis revealed a significant interaction between task condition and distractor compatibility $[F(2,30)=$ $4.03, p<.05]$. As can be seen in Figure 3, distractor interference effects were significantly reduced in the high-load in comparison with the low-load condition $[t(15)=2.2$, $p<.025$, for the difference] and the degraded low-load condition $[t(15)=2.08, p<.05$, for the difference $]$. There was no difference in the distractor interference effects between the conditions of low load and degraded low load $(p>.10)$. Distractor interference was significant in the conditions of low load $[M=36 \mathrm{msec}, t(15)=7.63, p<.001]$ and degraded low load $[M=43 \mathrm{msec}, t(15)=4.71, p<$ $.001]$, but not in that of high load $(M=12 \mathrm{msec}, p>.10)$.

In the error rates, there was also a significant interaction between task condition and distractor compatibility $[F(2,30)=21.64, p<.001]$. Distractor interference effects were significantly increased in the degraded low-load condition $(M=15 \%)$ in comparison with the low-load $[M=$ $2 \% ; t(15)=5.8, p<.001$, for the difference], and the highload $[M=3 \% ; t(15)=4.7, p<.001$, for the difference $]$ conditions.

Distractor facilitation effects. In order to establish whether compatible distractors produced any facilitation, ANOVA comparisons of RTs and error rates in compatible and neutral conditions between task conditions (low load, high load, degraded low load) were conducted. As can be seen in Figure 3, there were no main effects of compatibility and no interaction between task condition and distractor compatibility on RTs ( $F<1$ in all comparisons). The error rate analysis revealed a significant interaction $[F(2,30)=7.41, p<.01]$. This interaction showed that the significant facilitation from compatible (in comparison with neutral) distractors in the degraded low-load condition $[M=4 \% ; t(15)=2.38, p<.05]$ was significantly reduced in the high-load $[M=-1 \% ; t(15)=3.47, p<.01$, for the difference] and low-load $[M=0 \% ; t(15)=2.35, p<$ .05 , for the difference] conditions (see Figure 3 ).

In summary, once again the results showed that, in comparison with low perceptual load, both the manipulation of high perceptual load and that of target degradation led to an increase in task difficulty. Distractor processing, however, was reduced only by the manipulation of high perceptual load. In contrast, target-stimulus degradation increased the distractor effects on the error rates with a similar, although statistically nonsignificant, trend of increased distractor interference effects on RTs. The failure to find a significant increase in distractor effects on RTs may be attributed to the fact that distractors in the degraded low-load condition were presented for half the duration of distractor presentation in the other conditions.

Intersubject analysis of relative speed between task conditions. In terms of general task difficulty, although con- 
ditions of both high perceptual load and degraded low load increased RTs and error rates in comparison with those of low perceptual load, target degradation produced a greater increase in the error rates than did high load, whereas high load produced a greater slowing of responses than did degraded low load. However, inspection of the individual subjects' data revealed that, although the average overall RT in the degraded low-load condition was $50 \mathrm{msec}$ shorter than the average overall RT in the high-load condition, there was great variability in overall RTs in the degraded low-load condition. In fact, 5 of the subjects had overall RTs that were substantially longer in the degraded low-load condition than in the high-load condition.

In order to further examine whether the reduced distractor interference effects in the high-load condition versus the degraded low-load condition could be attributed to a difference in the speed between these conditions, we reanalyzed the distractor interference results with an additional factor of relative overall speed in these two conditions. Using a median split procedure, the subjects were divided into two groups according to whether their relative difference in speed between the degraded low-load condition and the high-load condition was higher or lower than the median difference in speed between these conditions across all subjects $(M d n=54 \mathrm{msec})$. The subjects in Group A were slower in the degraded low-load condition than in the high-load condition, whereas those in Group B were slower in the high-load condition than in the degraded low-load condition. Figure 4 presents the mean RTs and error rates for the two subject groups as a function of task condition and distractor compatibility.
As can be seen in Figure 4, although the two groups of subjects differed in their relative speeds between the task conditions, the pattern of distractor interference effects was the same for the two subject groups. This was confirmed in ANOVAs of the RTs and error rates, both of which showed no significant interaction between subject group, task condition (low load, high load, degraded low load), and distractor compatibility (incompatible, neutral; $F<1$ for both RTs and error rates). Thus, this analysis allows us to rule out relative processing speed between the conditions of high load and degraded low load as a way of accounting for the different distractor interference effects between these conditions.

\section{EXPERIMENT 3}

In Experiments 1 and 2, target stimuli in the degraded low-load conditions were different from those in the other task conditions. In Experiment 3, we attempted to generalize the effects of target-stimulus degradation to a new manipulation of stimulus degradation that did not involve any change in the target stimulus between the degraded low-load condition and the other conditions. This was achieved by manipulating the eccentricity of target position in each of the perceptual load conditions. Again, perceptual load was manipulated by varying the relevant set size from one to six letters, but, instead of being presented in circular arrays, target and relevant nontarget stimuli were now presented in six positions arranged in a vertical line, crossing at fixation. The two central positions were at $0.6^{\circ}$ eccentricity, the two intermediate positions on the

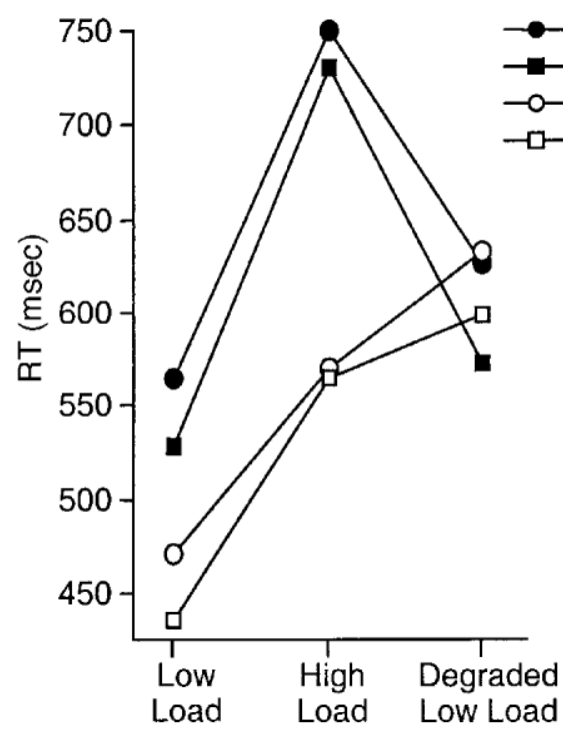

Group A - Incompatible
Group A - Neutral
Group B - Incompatible
Group B - Neutral

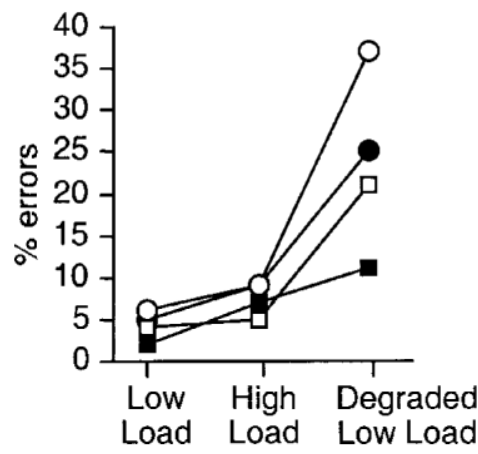

Task Condition

Figure 4. Mean correct-response reaction times (RTs) and error rates as a function of task condition, distractor compatibility, and relative speed in the degraded low-load versus high-load conditions in Experiment 2. The subjects in Group A $(n=8)$ had a greater-than-median speed difference between high load and degraded low load. Those in Group B $(n=8)$ had a smaller-than-median speed difference between high load and degraded low load. 


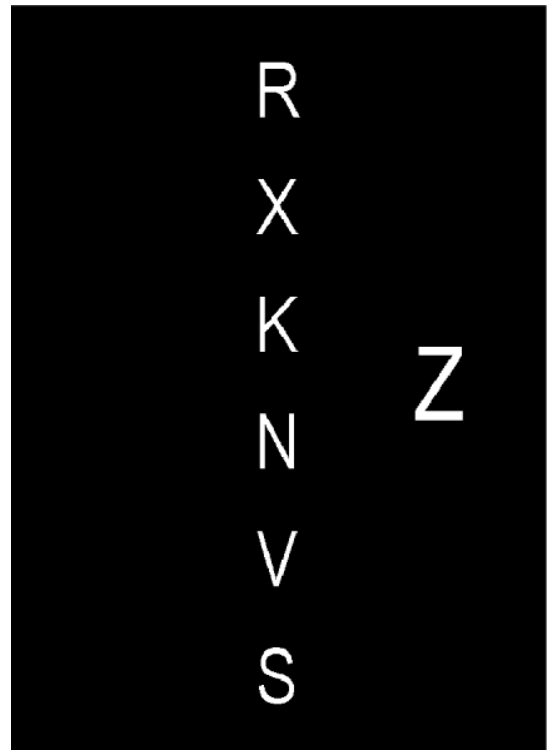

Figure 5. An example of a stimulus display in the condition of high load with target $(X)$ at $1.8^{\circ}$ eccentricity in Experiment 3. In the low-load condition, the nontargets were not present.

line were at $1.8^{\circ}$ eccentricity, and the end positions were at $3^{\circ}$ eccentricity (see Figure 5 for an example stimulus display). ${ }^{1}$ The exposure duration of the displays was $100 \mathrm{msec}$ (as in the previous experiments), thus ensuring that eye movements would be precluded and could not counteract our manipulation of retinal eccentricity.

Given that perceptual load and eccentricity were varied in an orthogonal design in this experiment, we could now also examine the nature of the interaction between perceptual load and target-stimulus degradation. Finally, target position in Experiment 3 was randomly intermixed within blocks, ruling out strategy differences in the effects of target degradation.

\section{Method}

Subjects and Apparatus. Sixteen students from University College London participated. All had normal or corrected-to-normal vision and were paid for their participation. None had participated in Experiment 1 or Experiment 2. The apparatus and viewing distance were the same as in Experiments 1 and 2.

Stimuli and Procedure. All the stimuli were presented in light gray (color 7 in the MEL palette) on a black background. Each display consisted of a target letter (either $\mathrm{Z}$ or $\mathrm{X}$ ) subtending $0.6^{\circ}$ of visual angle vertically $\times 0.4^{\circ}$ horizontally, and an irrelevant distractor letter subtending $0.9^{\circ}$ of visual angle vertically $\times 0.5^{\circ}$ horizontally. The distractor letter was presented on the left or right at a distance of $1.8^{\circ}$ from fixation (measured from the center of the letter). The target letter was presented at random in one of six possible locations arranged on a vertical line subtending $6.7^{\circ}$ from top to bottom and centered at fixation. On low-load trials, only the target letter was presented, and the other five locations remained empty. On highload trials, the other five locations were occupied by the nontarget letters $\mathrm{S}, \mathrm{N}, \mathrm{K}, \mathrm{V}$, and $\mathrm{R}$, which were of the same size as the target. The target and nontarget letters appeared equally often in each position, in a random order, and were separated from each other by $0.6^{\circ}$ from edge to edge. This resulted in eccentricities (measured from

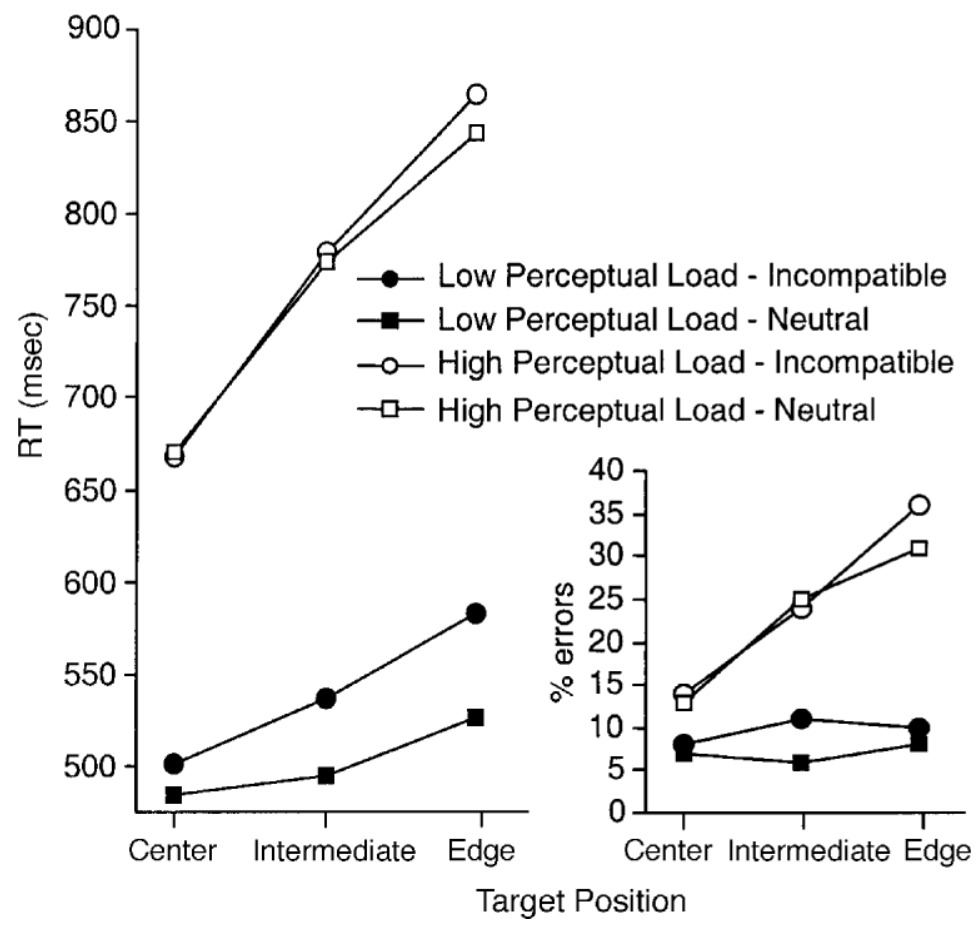

Figure 6. Mean correct-response reaction times (RTs) and error rates as a function of perceptual load, target position, and distractor compatibility in Experiment 3 . 
fixation to the center of the letter) of $0.6^{\circ}$ for the central letters, $1.8^{\circ}$ for the letters in the intermediate positions, and $3^{\circ}$ for the letters in the edge positions. The distractor was equally likely to be incompatible or neutral (the letter $\mathrm{P}$ ) with respect to the target responses. Compatible distractor conditions were not included in this experiment, because in the previous experiments the facilitation effects either were nonsignificant or followed the same pattern as did those of the distractor interference effects. The various combinations of target identity, target position, distractor identity, and distractor position occurred equally often in each block. For each of the load conditions, 72 different displays were created according to these specifications. The rest of the procedure was the same as in Experiment 1, except for the following changes. The subjects now responded by pressing " 1 " on the numeric computer keypad for Z targets and " 2 " for X targets. Half of the subjects performed four blocks of low load followed by four blocks of high load, and the other half performed in the opposite order. Each load condition was preceded by a short practice block of 20 trials.

\section{Results and Discussion}

Mean RTs and error rates were calculated for each subject as a function of the experimental factors (see Figure 6). Trials with incorrect responses or RTs over 2 sec were excluded from analysis. Fewer than $0.5 \%$ of the trials were excluded due to the 2 -sec cut-off criterion.

Effects of perceptual load and stimulus degradation on task difficulty. Within-subjects ANOVAs of the RTs and error rates with the factors of perceptual load (low, high), target position (center, intermediate, edge), and distractor compatibility (incompatible, neutral) revealed a main effect of perceptual load on RTs $[F(1,15)=$ $85.91, p<.001]$ and error rates $[F(1,15)=147.45, p<$ $.001]$. RTs in the high-load condition $(M=767 \mathrm{msec})$ were longer than RTs in the low-load condition $(M=$ $521 \mathrm{msec})$, and error rates in the high-load condition $(M=$ $24 \%)$ were higher than those in the low-load condition $(M=8 \%)$. There was also a main effect of target position on RTs $[F(2,30)=50.01, p<.001]$ and error rates $[F(2,30)=$ $56.85, p<.001]$. RTs to targets in the edge positions $(M=$ $705 \mathrm{msec}$ ) were longer than those to targets in the intermediate positions $[M=647 \mathrm{msec} ; t(15)=7.32, p<.001$, for the difference], which, in turn, resulted in longer RTs in comparison with targets in the center positions $[M=$ $581 \mathrm{msec} ; t(15)=5.49, p<.001$, for the difference]. Error rates followed the same pattern and were higher for targets in the edge positions $(M=21 \%)$ than for those in the intermediate positions $[M=16 \% ; \mathrm{t}(15)=5.59, p<.001$, for the difference], which, in turn, resulted in more errors than did targets in the center positions $[M=10 \% ; t(15)=5.32$, $p<.001$, for the difference]. Thus, both the manipulation of perceptual load and that of target position were successful in increasing task difficulty.

There was also an interaction between perceptual load and target position in both the RTs $[F(2,30)=23.77, p<$ $.001]$ and the error rates $[F(2,30)=42.9, p<.001]$. The increases in RTs and in error rates as a function of eccentricity were more pronounced in conditions of high perceptual load than in those of low perceptual load. This result can be explained by an interaction of eccentricity and an additional effect of crowding produced by the highload letters in this experiment. The extent to which neigh- boring stimuli produce crowding effects and, thus, interfere with target perception (also known as lateral masking) typically increases with increased eccentricity (see Bouma, 1970). The present experiment involved targets at more eccentric positions than those used in the previous experiments (recall that the circular arrays in the previous experiments were presented at $1.3^{\circ}$ from fixation); thus, the additional letters in the high-load condition may have produced crowding, and these crowding effects were greater for targets in more eccentric positions.

Distractor interference effects. Our main interest, however, was in the interactive effects of perceptual load and target position on distractor processing. The ANOVA of RTs revealed a significant interaction between perceptual load and distractor compatibility $[F(1,15)=8.23, p<$ $.02]$. As in the previous experiments, the distractor effects in the low-load condition $[M=39 \mathrm{msec}, t(15)=7.25, p<$ $.001]$ were significantly reduced by high load $(M=$ $8 \mathrm{msec} ; p>.30$; see Figure 6). There was also an interaction between target position and distractor compatibility $[F(2,30)=4.62, p<.02]$. As we predicted, increased eccentricity of target position led to increased distractor effects (see Figure 5). Distractor interference effects were significantly greater for targets in the edge positions $(M=$ $39 \mathrm{msec}$ ) than for those in the intermediate [ $M=24 \mathrm{msec}$; $t(15)=1.85, p<.05$, for the difference $]$ and center $[M=$ $8 \mathrm{msec} ; t(15)=2.95, p<.005$, for the difference] positions. The difference between distractor interference in the intermediate and center positions did not reach significance $[t(15)=1.36, p=.097]$. There was no three-way interaction between perceptual load, target position, and distractor compatibility $(F<1)$. There were no significant interactions with distractor compatibility in the ANOVA of error rates $[F(2,30)=2.56, p>.09$ for the three-way interaction; $F<1$ for the two-way interactions].

Thus, despite the similar increase in task difficulty for both manipulations of perceptual load and those of target degradation, these again had opposite effects on distractor processing. Whereas an increase in perceptual load led to a reduction in distractor interference, the manipulation of target-stimulus degradation led to increased distractor processing.

\section{GENERAL DISCUSSION}

In this study, it has been demonstrated that processing of irrelevant distractors depends on the type of processing demands imposed by a relevant task. Increased perceptual load in a relevant task induced by a demand to process a greater number of relevant stimuli resulted in decreased processing of irrelevant distractors. In contrast, increasing the difficulty of the relevant task by degrading the sensory quality of the target stimulus through reduced size and contrast (Experiment 1), through reduced contrast and presentation duration (Experiment 2), or through reduced retinal acuity in more eccentric positions (Experiment 3 ) has led to increased processing of irrelevant distractors in each case.

The contrast between the effects of perceptual load and sensory degradation of the target stimulus on distractor 
processing allows us to distinguish the effects of perceptual load from general effects of task difficulty and processing speed. Although both manipulations of perceptual load and those of target-stimulus degradation resulted in an increase of RTs and an increased number of errors (in comparison with a low-load condition with intact target stimuli), they had opposite effects on distractor processing. Thus, it is clear that the reduction in distractor effects seen under high perceptual load cannot simply be attributed to a general increase in task difficulty and the associated increase of RTs, but is, rather, more specific to the type of processing demands that are imposed by the relevant task.

In the conditions of high perceptual load, the target had to be searched for among other nontarget letters that were similar to it, and this clearly imposes a greater demand on attention (see, e.g., Duncan \& Humphreys, 1989; Treisman, 1991). The finding that distractor processing was reduced under these conditions concurs with previous findings showing that distractor effects are typically reduced in conditions of high perceptual load (e.g., Lavie, 1995; Lavie \& Cox, 1997; Lavie \& Fox, 2000; Rees et al., 1997) and provides support for the hypothesis that distractor processing depends on the extent to which the relevant processing leaves spare capacity that "spills over" to processing of irrelevant distractors (see Lavie, 1995, 2000, 2001).

In the condition of degraded low load, the target letter appeared alone in a central position and, thus, received full attention. Nevertheless, degradation of the target stimulus resulted in a marked increase in task difficulty, suggesting that allocation of full attention could not compensate for the sensory limits induced by stimulus degradation. This is exactly what we predicted from the perceptualload model, with the additional assumption that a manipulation of sensory degradation of the target stimulus should produce data limits rather than resource limits, and, therefore, that the increase in task difficulty produced by degrading the input for that task cannot be compensated for by applying more attentional resources to target processing (Norman \& Bobrow, 1975). The finding that distractor effects tended to increase in the degraded lowload conditions is expected in some models of attention. If the main effect of target degradation is to prolong processing time without affecting the availability of attention, then this manipulation provides a larger time window for distractor intrusions (C. W. Eriksen \& Schultz, 1979; Navon, 1989).

Although in some studies (e.g., Miller, 1991) it has been found that distractor effects tend to dissipate in conditions that produce longer RTs, this was clearly not the case in our study. Instead, our study emphasizes the importance of the nature of the process that led to an increase of RTs. Whereas perceptual load reduced distractor effects, a similar increase of RTs produced by stimulus degradation of the targets in fact increased distractor effects.

Another possible contribution to the increase in distractor effects in the degraded low-load conditions versus in the low-load conditions with intact targets may have been made by the effects of target degradation on the relative visual saliency of the distractor stimulus in comparison with that of the target stimulus. Although we did not directly manipulate the visual quality of the distractor stimulus in our experiments, our manipulations of degradation of the target stimulus may have varied the relative visual quality of the distractor stimulus in comparison with that of the target stimulus. Thus, the distractor stimulus should have benefited from better visual acuity (and was therefore more visually salient) than the target in the low-load conditions with degraded targets (e.g., stimulus contrast of the distractor with the background was greater than that of the degraded targets with the background in Experiments 1 and 2). However, the visual quality of the distractor and target stimuli was fairly similar in the conditions with intact targets. As a result, the more salient distractors may have been better able to capture attention and produce more interference in the conditions of degraded low load than in the conditions of low load with intact target stimuli. Note that the relative visual saliency of target versus distractor stimuli may also explain the increased priming effects from distractors reported in previous studies in which the visual acuity of the targets was reduced (e.g., Kane, May, Hasher, Rahhal, \& Stoltzfus, 1997).

It is important to note, however, that the relative saliency of the distractor versus that of the target cannot explain why distractor effects were reduced in high load in comparison with low load, mainly for two reasons. First, visual saliency of the distractor relative to the target seemed also to be greater in the condition of high load than in that of low load, because in the high-load condition the target was more "hidden" among the relevant nontarget letters, and the distractor appeared on its own in the periphery, thus forming an attention-capturing singleton. In the low-load condition, however, the target was presented alone in a central position and was, thus, at least as salient as the distractor. Nevertheless, distractor effects were significantly smaller in the conditions of high load than in those of low load. Second, in Experiment 3, perceptual load reduced distractor effects to the same extent in the different conditions of target eccentricity, which would also appear to vary in terms of the relative visual saliency of the distractors in comparison with the targets. In other words, the acuity and, therefore, the visual saliency of the distractors were greater in comparison with those of parafoveal and peripheral targets than in comparison with those of foveal targets (because the target in the more peripheral positions both had reduced acuity and was more vulnerable to crowding effects; see, e.g., Bouma, 1970), yet distractor effects were equally reduced by load in all target positions. Thus, although stimulus saliency of the distractor relative to the target may well have contributed to the finding that distractor effects are increased in the degraded low-load condition, it cannot account for the findings that perceptual load had an effect opposite to that of target degradationthat is, of reducing, rather than increasing, distractor processing.

We conclude that processing of irrelevant distractors does not depend on general task difficulty, but, rather, on 
the extent to which the relevant task specifically imposes high load on attention (Lavie, 1995, 2001; Lavie \& Cox, 1997; Lavie \& Fox, 2000). The present results clarify the notion of perceptual load by distinguishing it from general effects of task difficulty, and suggest that capacity limits (a concept that previously has typically been applied only to discussions of relevant task processing; for a review, see Lavie \& Tsal, 1994) play an important role in determining the processing of irrelevant distractors.

\section{REFERENCES}

Bonnel, A., Possamai, C. A., \& Schmitt, M. (1987). Early modulations of visual input: A study of attentional strategies. Quarterly Journal of Experimental Psychology, 39A, 757-776.

Bouma, M. (1970). Interaction effects in parafoveal letter recognition. Nature, 226, 177-178.

Broadbent, D. E. (1958). Perception and communication. London: Pergamon.

Deutsch, J. A., \& Deutsch, D. (1963). Attention: Some theoretical considerations. Psychological Review, 70, 80-90.

Deutsch, J. A., \& Deutsch, D. (1967). Comments on "Selective attention: Perception or response?" Quarterly Journal of Experimental Psychology, 19, 362-363.

Driver, J., \& TIPPER, S. P. (1989). On the nonselectivity of selective seeing: Contrasts between interference and priming in selective attention. Journal of Experimental Psychology: Human Perception \& Performance, 15, 304-314.

Duncan, J. (1980). The locus of interference in the perception of simultaneous stimuli. Psychological Review, 87, 272-300.

Duncan, J., \& Humphreys, G. W. (1989). Visual search and stimulus similarity. Psychological Review, 96, 433-458.

ERIKSEN, B. A., \& ERIKSEN, C. W. (1974). Effects of noise letters upon the identification of a target letter in a nonsearch task. Perception \& Psychophysics, 16, 143-149.

ERIKSEN, C. W., \& Schultz, D. W. (1979). Information processing in visual search: A continuous flow conception and experimental results. Perception \& Psychophysics, 25, 249-263.

Francolini, C. M., \& Egeth, H. E. (1980). On the nonautomaticity of "automatic" activation: Evidence of selective seeing. Perception \& Psychophysics, 27, 331-342.

Gatti, S. V., \& Egeth, H. E. (1978). Failure of spatial selectivity in vision. Bulletin of the Psychonomic Society, 11, 181-184.

Graham, N. V. S. (1989). Visual pattern analyzers. New York: Oxford University Press.

Huckauf, A., Heller, D., \& Nazir, T. A. (1999). Lateral masking: Limitations of the feature interaction account. Perception \& Psychophysics, 61, 177-189.

Kane, M. J., May, C. P., Hasher, L., Rahhal, T., \& Stoltzfus, E. R. (1997). Dual mechanisms of negative priming. Journal of Experimental Psychology: Human Perception \& Performance, 23, 632-650.

Lavie, N. (1995). Perceptual load as a necessary condition for selective attention. Journal of Experimental Psychology: Human Perception \& Performance, 21, 451-468.

LAVIE, N. (2000). Selective attention and cognitive control: Dissociating attentional functions through different types of load. In S. Monsell \& J. Driver (Eds.), Attention and performance XVIII (pp. 175-194). Cambridge, MA: MIT Press.
Lavie, N. (2001). The role of capacity limits in selective attention: Behavioral evidence and implications for neural activity. In J. Braun \& C. Koch (Eds.), Visual attention and cortical circuits (pp. 49-68). Cambridge, MA: MIT Press.

LAVIE, N., \& Cox, S. (1997). On the efficiency of attentional selection: Efficient visual search results in inefficient rejection of distraction. Psychological Science, 8, 395-398.

Lavie, N., \& Fox, E. (2000). The role of perceptual load in negative priming. Journal of Experimental Psychology: Human Perception \& Performance, 26, 1038-1052.

LaVIE, N., \& TSAL, Y. (1994). Perceptual load as a major determinant of the locus of selection in visual attention. Perception \& Psychophysics, 56, 183-197.

Miller, J. (1991). The flanker compatibility effect as a function of visual angle, attentional focus, visual transients, and perceptual load: A search for boundary conditions. Perception \& Psychophysics, 49, 270 288.

NAVON, D. (1989). The locus of attentional selection: Is it early, late, or neither? European Journal of Cognitive Psychology, 1, 47-68.

Norman, D. A., \& Bobrow, D. G. (1975). On data-limited and resourcelimited processes. Cognitive Psychology, 7, 44-64.

Rees, G., Frith, C., \& Lavie, N. (1997). Modulating irrelevant motion perception by varying attentional load in an unrelated task. Science, 278, 1616-1619.

SCHNEIDER, W. (1988). Micro Experimental Laboratory: An integrated system for IBM PC compatibles. Behavior Research Methods, Instruments, \& Computers, 20, 206-217.

Strasburger, H., Harvey, L. O., Jr., \& Rentschler, I. (1991). Contrast thresholds for identification of numeric characters in direct and eccentric view. Perception \& Psychophysics, 49, 495-508.

TIPPER, S. P. (1985). The negative priming effect: Inhibitory effects of ignored primes. Quarterly Journal of Experimental Psychology, 37A, 571-590.

Treisman, A. [M.] (1991). Search, similarity, and integration of features between and within dimensions. Journal of Experimental Psychology: Human Perception \& Performance, 17, 652-676.

Treisman, A. M., \& Geffen, G. (1967). Selective attention: Perception and response? Quarterly Journal of Experimental Psychology, 19, 1-18.

Treisman, A. M., \& Gelade, G. (1980). A feature-integration theory of attention. Cognitive Psychology, 12, 97-136.

UTTAL, W. R. (1987). The perception of dotted forms. Hillsdale, NJ: Erlbaum.

Wolford, G., \& Chambers, L. (1983). Lateral masking as a function of spacing. Perception \& Psychophysics, 33, 129-138.

YANTIS, S., \& Johnston, J. C. (1990). On the locus of visual selection: Evidence from focused attention tasks. Journal of Experimental Psychology: Human Perception \& Performance, 16, 135-149.

\section{NOTE}

1. Notice that each level of eccentricity combined different serial positions (e.g., letters with peripheral eccentricity were letters from the first and last positions, etc.), so that the effects of position eccentricity could not be due to effects of serial position in the vertical row. We thank John Palmer for raising this issue.

(Manuscript received May 2, 2001; revision accepted for publication September 6, 2002.) 